\title{
Deporte: modificaciones fisiológicas y evaluación para la prevención de la muerte súbita (Parte II). Corazón del deportista
}

\author{
Dres. Alejandro Cuesta ${ }^{1,2}$, Geraldine Rodríguez Estula 3 , Sergio Giovanetti4,5
}

\section{Resumen}

La práctica de deporte regular ha demostrado ser beneficiosa para la salud y prolongar la vida, tanto en personas sanas como en aquellas con patología cardiovascular (CV), a cualquier edad. También es verdad que aumenta el riesgo de morir en forma súbita. Recientemente, ha surgido evidencia de que el exceso puede ser nocivo y se está investigando. En los deportistas hay adaptaciones del aparato CV que pueden confundirse con anormalidades; por otra parte, existen patologías CV que progresan más rápido con el ejercicio y en las personas con alteraciones asintomáticas, el ejercicio puede desencadenar eventos adversos. La valoración predeportiva ha demostrado prevenir la muerte súbita. Para no efectuar diagnósticos erróneos que afecten la vida de los deportistas, es necesario conocer las adaptaciones clínicas, electrocardiográficas y anatómicas que se consideran normales y usar pautas precisas internacionales. En esta segunda entrega se valoran los aspectos relacionados al corazón del deportista, profundizando en el diagnóstico diferencial entre adaptación fisiológica y patología.

Palabras clave: DEPORTE

VALORACIÓN PREDEPORTIVA

CORAZÓN DE ATLETA

\section{Sport: physiological modifications and evaluation for the prevention of sudden death (Part II). Athlete's heart}

\section{Summary}

The practice of regular sports has proven to be beneficial for health, and prolong life, both in healthy people and in those with cardiovascular disease, at any age. It is also true that it increases the risk of dying suddenly. Evidence has recently emerged that excess could be harmful and is being investigated. In athletes there are adaptations of the cardiovascular apparatus, which can be confused with abnormalities; on the other hand, there are cardiovascular pathologies that progress faster with exercise, and in people with asymptomatic disorders, exercise can trigger adverse events. Pre-sport assessment has been shown to prevent sudden death. In order not to make erroneous diagnoses that affect the lives of athletes, it is necessary to know the clinical, electrocardiographic and anatomical adaptations that are considered normal and to use precise international guidelines. In this second installment, the aspects related to the athlete's heart will be evaluated, delving into the differential diagnosis between physiological adaptation and pathology.

Key words: $\quad$ SPORT

PRE-SPORT ASSESSMENT

ATHLETE`S HEART

1. Centro Cardiovascular Universitario, Hospital de Clínicas. Montevideo, Uruguay.

2. Instituto de Cardiología Integral, Servicio de Arritmias MUCAM. Montevideo, Uruguay.

3. Poder Judicial. PREMUDE, Gol al Futuro, Secretaría Nacional de Deporte. Montevideo, Uruguay.

4. Servicio Médico Integral (SMI). Montevideo, Uruguay.

5. Asociación Española. Montevideo, Uruguay.

Los autores declaran no tener conflictos de intereses.

Recibido Set 10, 2019; aceptado Ago 20, 2020 


\section{Esporte: modificações fisiológicas e avaliação para prevenção de morte súbita (Parte II). Coração do esportista}

\section{Resumo}

A prática de esportes regulares tem se mostrado benéfica para a saúde e prolonga a vida, em pessoas saudáveis e com doenças cardiovasculares, em qualquer idade. Também é verdade que aumenta o risco de morrer repentinamente. Recentemente, surgiram evidências de que o excesso pode ser prejudicial e estão sendo investigadas. Nos atletas há adaptações do aparelho cardiovascular que podem ser confundidas com anormalidades; por outro lado, existem patologias cardiovasculares que progridem mais rapidamente com o exercício e em pessoas com distúrbios assintomáticos, o exercício pode desencadear eventos adversos. A avaliação pré-esporte demonstrou prevenir a morte súbita. Para não se fazer diagnósticos errôneos que afetam a vida dos atletas é necessário conhecer as adaptações clínicas, eletrocardiográficas e anatômicas consideradas normais e utilizar diretrizes internacionais precisas. Nesta segunda edição, serão avaliados os aspectos relacionados ao coração do atleta, aprofundando-se no diagnóstico diferencial entre adaptação fisiológica e patologia.

Palavras chave: $\quad$ ESPORTE

AVALIAÇAO PRÉ-ESPORTES

CORAÇAO DO ESPORTISTA

\section{Normalidad}

Para buscar y encontrar alteraciones y factores de riesgo de muerte súbita (MS) en los deportistas, es imprescindible conocer las variantes fisiológicas normales de esta población.

El ejercicio aumenta las necesidades metabólicas, fundamentalmente el consumo de oxígeno y nutrientes y la eliminación de dióxido de carbono $\left(\mathrm{CO}_{2}\right)$ y ácido láctico. Desde el punto de vista hemodinámico, estas demandas son satisfechas por la capacidad de aumentar el gasto cardíaco. En personas bien entrenadas, el gasto puede alcanzar valores pico de 35-40 l/min, es decir, 8-10 veces el volumen /minuto basal. Para lograrlo, aumenta la contractilidad del miocardio, la frecuencia cardíaca y disminuyen las resistencias periféricas. El gran incremento del gasto cardíaco durante el ejercicio, a pesar de la disminución de las resistencias periféricas, determina un aumento de la presión arterial sistólica, con mantenimiento o descenso de las cifras de presión diastólica ${ }^{(1)}$.

Para alcanzar este rendimiento, se debe realizar un entrenamiento adecuado. El entrenamiento efectuado en forma crónica y sistemática determina modificaciones del aparato cardiovascular (CV) y su sistema de regulación, que se evidencian posteriormente en el reposo. A estos cambios fisiológicos, estructurales y eléctricos, se les denomina "corazón del deportista". Para que se produzcan, se requiere de un entrenamiento intenso, frecuente, durante un tiempo prolongado que implique la utilización de gran parte del cuerpo ${ }^{(2)}$. Obviamente, no es esperable una hipertrofia ventricular del deportista en sujetos que hacen actividad esporádica o que practican un deporte como la pesca.

El ejercicio puede ser clasificado en dos tipos: dinámico y estático. Los ejercicios dinámicos involu- cran gran movimiento de las articulaciones y cambios de la longitud muscular, pero poca fuerza intramuscular. Suponen un metabolismo aeróbico importante y por ello también se los denomina aeróbicos. Los ejercicios estáticos suponen una fuerza intensa con poco o ningún cambio en la longitud muscular. Son realizados mediante la producción explosiva de energía de forma anaeróbica. Prácticamente todos los deportes tienen alguna proporción de ambos tipos de ejercicio. En base a ello, en 1994, se propuso una clasificación aún vigente de todos los deportes. Se dividieron en nueve tipos de acuerdo al nivel (bajo, medio o alto) de cada componente ${ }^{(3)}$. El fútbol, por ejemplo, tiene un nivel dinámico alto y estático bajo, como el tenis. El rugby es moderadamente dinámico y moderadamente estático, como las carreras de velocidad. El levantamiento de pesas y el judo son muy estáticos y poco dinámicos ${ }^{(1-3)}$. El tiro o el bowling tienen niveles bajos de ambos componentes y nunca encontraremos en sus jugadores adaptaciones cardíacas.

Por ser el deporte más practicado en Uruguay, profundizaremos en el fútbol. Es un deporte de características acíclicas. Los deportes acíclicos se caracterizan por la organización de diferentes acciones motrices complejas y trabajo intenso, realizado en poco tiempo de competencia; involucra también diversidad de acciones técnicas. Lo mismo ocurre en básquetbol y voléibol. En contraposición, los deportes cíclicos se caracterizan por seguir un mismo patrón de movimiento de manera constante, como es el caso de la natación, remo y ciclismo.

La actividad física promedio del jugador de fútbol se puede distribuir en cinco niveles de intensidad con diferentes porcentajes de tiempo en cada uno, durante un partido de 90 minutos. El 40\% del tiempo está caminando, 35\% trotando, $17 \%$ estático 
de pie, $8 \%$ en carrera de alta velocidad y menos de $1 \%$ en sprint ${ }^{(1)}$. Pero en el fútbol existen diferentes posiciones, roles, que tienen patrones de ejercicio diferentes. El arquero realiza acciones anaeróbicas explosivas y aeróbicas de baja intensidad, y recorre una distancia de aproximadamente cuatro kilómetros por partido. Los defensores y los delanteros recorren distancias mayores, 6-8 kilómetros. Tienen también reacciones explosivas, pero las aeróbicas son de moderada intensidad. Los volantes tienen un trabajo aeróbico de moderada-alta intensidad, recorriendo distancias que superan los 10 kilómetros por partido ${ }^{(1)}$

Los cambios en el corazón del deportista se pueden clasificar arbitrariamente en clínicos, morfológicas y eléctricos. La magnitud de éstos depende del tipo de deporte y de la intensidad con la que se practique. Pero estos factores no alcanzan para explicar las diferencias que existen entre deportistas de igual grupo etario, competencia y nivel de entrenamiento, lo que induce a pensar que existen factores genéticos involucrados en su aparición. Otro aspecto a considerar es la heterogeneidad entre razas.

\section{Cambios clínicos}

Las particularidades clínicas que se encuentran en los deportistas son secundarias a las anatómicas y eléctricas. Es importante conocerlas para que no llamen la atención y conduzcan a la solicitud inadecuada de exámenes.

Cuando el deportista es delgado y dependiendo de la morfología del tórax, se puede palpar la punta cardíaca como un latido intenso por fuera de la línea de referencia. Junto a ello un pulso periférico lento y amplio, aunque sin aumento de la presión diferencial.

A nivel central, es posible auscultar soplos en foco pulmonar y mesocardio. Estos tienen siempre características denominadas funcionales. Se auscultan al inicio de la sístole, respetan ambos ruidos, no tienen irradiaciones y se modifican y hasta desaparecen con las variaciones del retorno venoso y la frecuencia cardíaca, que se logran con la maniobra de Valsalva, al ponerse en cuclillas o de pie bruscamente, así como con inspiración y espiración profundas.

\subsection{Cambios eléctricos}

Para identificar un electrocardiograma (ECG) anormal que presente signos de enfermedad o de factores de riesgo de MS, debemos conocer el ECG normal del deportista. Este varía de acuerdo a la edad, raza y deporte que se practique. El entrenamiento determina cambios importantes a nivel del sistema nervioso autónomo ${ }^{(4)}$, y es común encon- trarlos en personas que han sido deportistas en el pasado. Existe un incremento del tono vagal en reposo, disminución del tono simpático y menor nivel de catecolaminas circulantes, a lo que se agrega una desensibilización de los efectores vasculares al estímulo adrenérgico. También pueden producirse cambios electrofisiológicos a nivel de los canales iónicos y en el nódulo sinusal ${ }^{(5,6)}$.

Por lo anterior, disminuye la frecuencia cardíaca en reposo y ante esfuerzos submáximos. La frecuencia cardíaca máxima, sin embargo, no se modifica y está estrechamente relacionada con la edad. La bradicardia se encuentra presente en la gran mayoría de los deportistas entrenados; está influida por el grado de entrenamiento y probablemente por factores constitucionales heredados y es de mayor magnitud en los deportes con mayor componente dinámico o aeróbicos ${ }^{(2)}$.

La frecuencia cardíaca de reposo que actualmente se toma como límite mínimo normal en personas entrenadas es de $30 \mathrm{cpm}$. Las pausas que superen los 2,5 segundos se consideran anormales ${ }^{(7)}$.

Se conserva la variación cíclica respiratoria, que puede ser de tal entidad que se confunda a la auscultación o en un trazado breve de ECG, con una extrasistolía auricular perisinusal.

La frecuencia sinusal puede ser por momentos superada por la frecuencia del automatismo del nódulo auriculoventricular o incluso de otros focos auriculares. En el primer caso, ritmo nodal, en el segundo ritmo auricular. Ambas situaciones son normales en la medida que ocurran en el reposo y que desaparezcan con la actividad, con el consiguiente aumento de la frecuencia sinusal. Pueden competir ambos ritmos y ello, si bien crea problemas diagnósticos, debe considerarse normal.

El tono autonómico y las modificaciones eléctricas del miocárdico específico no solo son evidentes en la generación del impulso, sino también en la conducción. Es así que pueden aparecer cambios en la despolarización auricular, en la unión auriculoventricular y más raramente a nivel ventricular. No se han notificado cambios en la conducción sino-auricular.

Es relativamente común el intervalo $\mathrm{PR}$ mayor de $200 \mathrm{~ms}$, es decir, el bloqueo auriculoventricular de primer grado con frecuencias bajas, en reposo, que desaparece al aumentar la frecuencia. Si no desaparece, se debe pensar en anormalidad(8). Es más frecuente por la noche y se detecta en el Holter.

El bloqueo auriculoventricular de segundo grado Mobitz 1 también puede ser una consecuencia normal del entrenamiento. Meytes y colaboradores, en 1975, realizaron un estudio con ECG basal a 126 atletas de alto rendimiento, 11 de los cuales presen- 
taron bloqueo auriculoventricular de primer grado y en tres se encontró el fenómeno de Wenckebach. Esto aparecía luego de al menos 15 minutos de descanso en posición reclinada y desaparecía al sentarse o ponerse de pie. Luego de seis años de seguimiento se vio que el fenómeno era solo detectable durante las temporadas de entrenamiento intensivo y no aparecía luego de pocas semanas de su finalización. No hubo enfermedades cardíacas o disminución del rendimiento durante ese período ${ }^{(9)}$. En los deportistas con Mobitz 1, siempre se demuestra en algún momento bloqueo auriculoventricular de primer grado confirmando el mecanismo extrínseco, es decir, tono vagal elevado.

El bloqueo auriculoventricular Mobitz 2 es menos común. Ha sido descrito en deportistas normales, pero siempre obliga a una investigación más profunda ${ }^{(10)}$. El bloqueo auriculoventricular completo es una eventualidad muy rara, siempre debe estudiarse y probablemente desentrenar al deportista antes de plantear otro tipo de estrategias ${ }^{(10,11)}$.

Los trastornos de la conducción ventricular que determinan una prolongación del QRS > 120 ms no están asociados habitualmente a la práctica de deporte y en principio deben considerarse patológicos. Pueden aparecer en frecuencia similar a la población general normal, pero deben siempre estudiarse. No hay diferencia para el bloqueo de rama derecha o izquierda.

Es relativamente común la aparición de ondas $\mathrm{R}$ ' en derivaciones precordiales derechas con $\mathrm{QRS}$ de duración normal. Se refiere como bloqueo incompleto de rama derecha. Algunos autores dudan de la existencia de dicho trastorno de conducción, y lo asignan a la hipertrofia o sobrecarga del ventrículo derecho. También se ha adjudicado a la persistencia del patrón juvenil, a dilatación aguda del ventrículo derecho, a infarto posterior o deformaciones esqueléticas. Se sabe que ocurre en $2,4 \%$ de la población no deportista sana y en 12\%-32\% de deportistas sin patología $^{(12-14)}$. El tema no está bien definido y, en estos casos, podría coexistir más de un mecanismo: enlentecimiento de la conducción en el tracto de salida del ventrículo derecho y aumento de la masa ventricular por la sobrecarga. No es motivo de limitación para el deporte. Si se asocia clínicamente a desdoblamiento fijo del segundo ruido, debe descartarse una comunicación interauricular.

El patrón de bloqueo incompleto de rama derecha se puede observar también en pacientes con displasia arritmogénica del ventrículo derecho (DAVD), asociado habitualmente a otras anormalidades: final lento o mellado del QRS (onda épsilon), o ambos, inversión de onda T en precordiales más allá de V2, voltajes bajos en derivaciones de los miembros y ex- trasístoles ventriculares con morfología de bloqueo de rama izquierda. También puede confundirse con un patrón de Brugada, caracterizado por un punto J de inicio alto que luego desciende, supra-convexo y se continúa con una onda $\mathrm{T}$ negativa en al menos una derivación entre V1-V3.

La amplitud de voltaje de las ondas del QRS en el deportista es un problema importante y una de las principales causas de falsos positivos.

Sharma y colaboradores estudiaron 1.000 atletas de élite entre 14 y 18 años y los compararon con 300 controles no atléticos emparejados por sexo, edad y área de superficie corporal ${ }^{(13)}$. Los deportistas tuvieron una duración del intervalo PR, del QRS y el QT significativamente más prolongada: 153 vs 140, 92 vs 89 y 391 vs 379 milisegundos, respectivamente. El criterio de voltaje de Sokolow para hipertrofia ventricular izquierda ( $\mathrm{R}$ en V1 + S en V5 o V6 $>35 \mathrm{~mm}$ ) fue más común en atletas (45\% vs $23 \%$ ), pero ninguno de ellos tenía desviación del eje a izquierda, depresión del segmento ST, inversión de la onda T profunda u ondas Q patológicas ${ }^{(8)}$. El criterio de hipertrofia ventricular izquierda, cuando ésta constituye una modificación adaptativa, no se asocia a criterios de sobrecarga auricular izquierda. Ante estas asociaciones debe pensarse en patología $^{(14)}$.

En cuanto a la repolarización ventricular, el patrón denominado repolarización precoz es muy común en deportistas. En su definición clásica se caracteriza por un supradesnivel del punto J desde el cual asciende rápidamente la onda T. Hay entonces una elevación cóncava del segmento ST, más frecuente en las derivaciones precordiales. Se han definido subtipos, dependiendo del grado de elevación del punto J, si hay una onda final llamada onda $\mathrm{J}$ o si el QRS se "funde" con el ST a través de un segmento "empastado"(15). Esta complejidad deviene de algunos autores que en estudios epidemiológicos de gran porte han podido demostrar una leve asociación entre repolarización precoz y MS en pacientes con corazón normal(16).

En el trabajo de Sharma y colaboradores, previamente mencionado, la elevación del segmento ST fue más común en los atletas que en los no atletas ( $43 \%$ vs $24 \%$; p <0,0001). Por el contrario, la inversión de la onda T en V2 y V3 estaba presente solo en el $4 \%$ de los deportistas, igual que en no atle$\operatorname{tas}^{(13)}$. Plantearon entonces que su presencia no podía considerarse una modificación normal del deportista sin una valoración más profunda.

La repolarización precoz es aun más frecuente en atletas afrodescendientes, se ha reportado entre 63\%-91\%(14). En ellos también se puede observar otra variación, que consiste en la elevación del ST 
convexo en las derivaciones V1 y eventualmente hasta $\mathrm{V} 4$, seguido de $\mathrm{T}$ invertida, presente en hasta $13 \%$ de los atletas afroamericanos. Para Drezner y colaboradores, en ausencia de síntomas y con examen físico normal, no se requiere más evaluación ${ }^{(14,17)}$. Si las ondas T invertidas superan V4, es prudente seguir evaluando al sujeto.

Un escenario totalmente distinto es cuando se observan alteraciones y particularmente inversiones de la onda $\mathrm{T}$ en derivaciones precordiales izquierdas. Estas deben considerarse siempre anormales y en especial sugestivas de miocardiopatía hipertrófica $(\mathrm{MCH})$.

\section{Criterios diagnósticos de electrocardiograma anormal}

En el año 2010, la Sociedad Europea de Cardiología (ESC), junto a la Asociación Europea para la Prevención y Rehabilitación Cardiovascular, presentaron una actualización de los criterios electrocardiográficos ${ }^{(18)}$. Clasificaron las "anormalidades" que pueden aparecer en los atletas en dos grupos: común y relacionada con la capacitación (grupo 1) o poco común y sin vinculación con el entrenamiento (grupo 2). Esta clasificación se basó en la prevalencia, la relación con el entrenamiento físico y la asociación con aumento del riesgo CV. Estableció de forma arbitraria una prevalencia menor de $5 \%$ para considerar una modificación como rara. Respecto a la pauta de 2005, quitó la bradicardia sinusal, el bloqueo auriculoventricular de primer grado y las modificaciones en la amplitud del QRS como criterios de alarma y los ubicó en el grupo 1. Consideró la repolarización precoz y la incluyó también en el grupo 1. Otro aspecto novedoso es que incluyó recomendaciones de cómo proseguir el estudio para cada hallazgo anormal particular.

En el año 2013, se presentaron los denominados Criterios de Seattle ${ }^{(17)}$, que surgieron de un consenso de expertos provenientes de la Sociedad Americana de Medicina del Deporte, la Asociación Europea para la Prevención y Rehabilitación Cardiovascular, el Centro de Evaluación e Investigación Médica de la Federación Internacional de Asociaciones de Fútbol y la Sociedad de Electrofisiología Pediátrica y Congénita. La lista se confeccionó mediante la evaluación de la bibliografía y no devino de un estudio epidemiológico específico. No incluye observaciones de sensibilidad ni especificidad de los distintos criterios. Se detallan en la tabla 1 .

Estos criterios incluyeron a las alteraciones del ritmo cardíaco y mejoraron su definición. También establecen de forma categórica la diferenciación racial que antes estaba solo mencionada en el texto ${ }^{(19)}$.
Tabla 1. Criterios de Seattle.

- Inversión de la onda T: $>1 \mathrm{~mm}$ de profundidad en dos o más de las siguientes derivaciones: V2-V6.

- Depresión del ST: $\geq 0,5 \mathrm{~mm}$ de profundidad en dos o más derivaciones.

- Ondas Q patológicas: > $3 \mathrm{~mm}$ de profundidad o > $40 \mathrm{~ms}$ de duración en dos o más derivaciones (a excepción de III y aVR).

- Bloqueo rama izquierda: $\mathrm{QRS} \geq 120 \mathrm{~ms}$.

- Retraso de conducción intraventricular: cualquier $\mathrm{QRS} \geq 140 \mathrm{~ms}$ de duración.

- Desviación del eje a izquierda $\left(-30^{\circ} /-90^{\circ}\right)$.

- Dilatación de aurícula izquierda: duración de onda $\mathrm{P}>120 \mathrm{~ms}$ en derivaciones I o II con porción negativa $\geq 1 \mathrm{~mm}$ y de $\geq 40 \mathrm{~ms}$ en V1.

Patrón de hipertrofia ventricular derecha: R-V1 + $\mathrm{S}-\mathrm{V} 5>10,5 \mathrm{~mm}$ y eje a derecha $>120^{\circ}$.

- Preexcitación ventricular: PR $<120$ ms con onda delta y QRS ancho (> $120 \mathrm{~ms}$ ).

- Intervalo QT largo: QTc $\geq 470 \mathrm{~ms}$ (masculino).

$\mathrm{QTc} \geq 480 \mathrm{~ms}$ (femenino).

QTe $\geq 500$ ms (prolongación QT marcada).

- Intervalo QT corto: QTc $\leq 320 \mathrm{~ms}$.

- Patrón de Brugada: segmento ST de origen alto.

- Bradicardia sinusal: $<30 \mathrm{cpm}$ o pausas sinusales $\geq$ $3 \mathrm{~s}$.

- Taquiarritmias auriculares: taquicardia supraventricular.

- Extrasístoles ventriculares: $\geq 2$ en 10 segundos de trazado.

- Arritmias ventriculares: duplas.

Con la aplicación de estos criterios mejoró sustancialmente la especificidad, que era el objetivo buscado.

Brosnan y colaboradores, en un estudio de 1.200 deportistas y de acuerdo a los criterios de la Sociedad Europea, encontraron 20\% con ECG anormal según las recomendaciones de la ESC y solo 0,3\% tenía efectivamente una anomalía cardíaca en una investigación posterior. Usando los Criterios de Seattle, el número de atletas clasificados como anormales descendió a 4,5\%. La mejora se debió a la reclasificación de atletas con un intervalo QTc equívoco, con inversión de onda T aislada a V1-V2 y con desviación aislada del eje a derecha o hipertrofia ventricular derecha por criterios de voltaje ${ }^{(20)}$. 
Tabla 2. Hallazgos electrocardiográficos normales en deportistas ${ }^{(17)}$.

- Bradicardia sinusal ( $\geq 30 \mathrm{cpm})$.

- Arritmia sinusal.

- Ritmo auricular ectópico.

- Ritmo de escape de unión.

- Bloqueo auriculoventricular de primer grado (intervalo PR > $200 \mathrm{~ms}$ ).

- Bloqueo auriculoventricular $2^{\circ}$ grado Mobitz tipo I (Wenckebach).

- Bloqueo incompleto de rama derecha.

Hipertrofia ventricular izquierda aislada por criterio de voltaje del QRS.

- Excepto cuando están asociados a: dilatación auricular izquierda; desviación del eje QRS a izquierda; depresión del segmento ST; inversión de onda T; onda $\mathrm{Q}$ patológica.

- Repolarización precoz (elevación del ST).

- Elevación del segmento ST supraconvexa combinada con inversión de onda $\mathrm{T}$ en las derivaciones V1-V4 en atleta afrodescendiente.

En el año 2014, un grupo de expertos europeos, liderado por el Dr. Sanjau Sharma (Universidad St. George's de Londres), publicó un estudio comparativo de los criterios de la ESC, los de Seattle y las modificaciones propuestas por el propio grupo de trabajo a las que denominaron "criterios refinados" y que se conocen como Criterios de Sharma. Consideraron que debían tener más en cuenta a los atletas no blancos ${ }^{(21)}$. Propusieron no catalogar como patológicos los siguientes diagnósticos electrocardiográficos aislados: 1) sobrecarga auricular izquierda, 2) sobrecarga auricular derecha, 3) desviación del eje del QRS a izquierda o 4) a derecha, 5) hipertrofia ventricular derecha. Recordemos que la hipertrofia ventricular izquierda aislada ya estaba descartada por los criterios europeos. Propusieron también: 6) no considerar patológica la inversión de onda $\mathrm{T}$ precedida por elevación convexa del segmento ST en V1-V4 en afrodescendientes. Cuando estuvieran presentes dos o más de estos seis patrones de forma simultánea, sí debían considerarse anormales y se continuaría con la evaluación. Mantuvieron el límite inferior de sospecha de QT largo en $\geq 470 \mathrm{~ms}$ en hombres $\mathrm{y} \geq 480 \mathrm{~ms}$ en mujeres ${ }^{(21)}$.

En el análisis retrospectivo de 5.500 deportistas con los criterios europeos ${ }^{(18)}$, encontraron $21,5 \%$ de ECG patológicos, con los de Seattle ${ }^{(22)} 9,6 \%$ y con los de Sharma 6,6\%(21). Los criterios "refinados" redujeron $37,5 \%$ los ECG anormales en atletas no blan- cos y $25,4 \%$ en blancos. La causa principal de anormalidad en no blancos continuó siendo la inversión de la onda $\mathrm{T}$ en las derivaciones inferiores y laterales. Luego aplicaron los tres criterios a una serie de 103 deportistas con $\mathrm{MCH}$, detectando a todos los portadores excepto dos casos que tenían ECG normal y habían sido diagnosticados por ecocardiograma y por el antecedente familiar ${ }^{(21)}$.

Un hecho que llama la atención es que en 58\% $(\mathrm{n}=3.210)$ de la población analizada para probar retrospectivamente los criterios, se había realizado ecocardiograma, porcentaje que excede largamente el de los ECG anormales y también la proporción de anormalidades clínicas informadas. Al parecer, la gran mayoría se hizo por política de la institución en la que practicaban. De los 3.210 deportistas estudiados con ecocardiograma, 40 (1,3\%) presentaron alguna anormalidad, siendo importante en 15 de ellos. Hubo cinco individuos con $\mathrm{MCH}$, cinco con Wolff-Parkinson-White, tres con QT largo, uno con Brugada y uno con origen anómalo coronario. Solo en uno de los 15 con anormalidades importantes, el ECG era normal, pero tenía síntomas. En ninguno de los 25 con anormalidades menores había signos electrocardiográficos de alarma.

\section{Cambios anatómicos}

El ejercicio genera fundamentalmente modificaciones cardíacas secundarias a sobrecarga de volumen. Ya mencionamos que en personas bien entrenadas el gasto puede alcanzar valores pico 8-10 veces el volumen/minuto basal normal. Los deportes con mayor componente aeróbico/dinámico generan más sobrecarga de volumen.

En el deportista entrenado, el hecho que más contribuye al incremento del volumen circulatorio es el aumento de la vascularización muscular. El aumento del número absoluto de capilares y de la relación capilares/miofibrillas se produce para recibir mayor cantidad de sangre circulante y disminuir la distancia fibra-capilar. Ello facilita el intercambio respiratorio y metabólico a las fibras musculares en activo, mejorando su disponibilidad de oxígeno y substratos energéticos ${ }^{(1)}$.

El manejo de mayor gasto y volumen lleva a un aumento del tamaño de las cavidades. El aumento del diámetro diastólico ventricular izquierdo conlleva a un volumen de eyección sistólico mayor, con un acortamiento menor y una pérdida inferior de energía por tensión y fricción. Es un mecanismo de compensación beneficioso. Los cambios morfológicos normales demostrados por ecocardiografía consisten en el aumento de las dimensiones de las cavi- 
dades y del grosor de las paredes. Lo que puede generar dudas es la extensión de esos cambios.

Pluim y colaboradores publicaron en el año 2000 un metaanálisis de trabajos que evaluaran parámetros anatómicos y funcionales ecocardiográficos en deportistas de distintas disciplinas y no deportistas controles. Las distintas disciplinas se clasificaron en: A) dinámicas (corredores de larga distancia), B) combinadas (ciclismo y remo) y C) estáticas (todas las que involucraban levantar o lanzar peso). No está incluido el fútbol, que correspondería al grupo B, y solo se incluyeron estudios con deportistas mayores de 18 años ${ }^{(23)}$.

Tanto el espesor de la pared posterior y el septum como el diámetro diastólico, el espesor parietal relativo y la masa ventricular izquierda, fueron significativamente menores en el grupo control, comparados con las disciplinas A, B y C; y menor en el grupo A comparado con $\mathrm{B}$ y $\mathrm{C}$, excepto en el caso del diámetro diastólico (menor en el grupo $\mathrm{C}$ ).

No hubo diferencia en la función ventricular sistólica evaluada en reposo por fracción de eyección y de acortamiento, ni en la función diastólica evaluada por la relación E/A.

Este estudio confirma muchos aspectos importantes. Primero, que la remodelación es una hipertrofia excéntrica, cuando hay hipertrofia, hay también dilatación. Segundo, que los deportes a predominio aeróbicos tienden a dilatar un poco más y los isométricos a la hipertrofia, pero no hay una diferencia detectable entre los deportes con ambos componentes y los isométricos puros. Tercero y más importante, que los cambios, aunque significativos, mantienen medidas medias en los límites superiores de los rangos considerados normales ${ }^{(23)}$.

Como en la población general, también hay situaciones que se alejan de la media y deben considerarse. Spirito y colaboradores realizaron ecocardiogramas a 1.000 deportistas de élite de 27 deportes distintos $^{(24)}$. El $38 \%$ tenía el diámetro diastólico final por encima del límite considerado normal (54 $\mathrm{mm}$ ) y en algún caso se llegó hasta los $66 \mathrm{~mm}$. Solo $2 \%$ tuvo un grosor parietal mayor al límite máximo normal (12 mm), y en un caso se llegó a 16 mm. Para estos autores, los atletas que se entrenan en deportes que se asocian a mayores dimensiones diastólicas de la cavidad ventricular izquierda también tienen mayor grosor parietal. Los atletas que se entrenan en deportes isométricos, como levantamiento de pesas y lucha libre, presentan valores altos de grosor de la pared ventricular en relación con la dimensión de la cavidad, pero el grosor absoluto de la pared se mantiene dentro de los límites normales ${ }^{(24)}$.
La diferenciación más compleja es entre las adaptaciones del deportista que se alejan mucho de la media y la MCH. El criterio clínico es muy importante. Los deportistas con grandes remodelaciones normales son sujetos entrenados de manera intensa y por largo tiempo y prácticamente todos hombres. Los sujetos con $\mathrm{MCH}$ pueden tener el antecedente familiar de la enfermedad o de un evento adverso.

Caselli y colaboradores realizaron un estudio comparativo entre deportistas y pacientes con MCH diagnosticada ${ }^{(25)}$. Eligieron 28 atletas de elite (grupo A), evaluados para ir a los juegos olímpicos de Beijing o a los juegos Pan-mediterráneos de Pescara, mayores de 18 años, con espesor parietal entre 13-15 milímetros, rango que consideraron la "zona gris". Aceptaron que eran jóvenes con hipertrofia fisiológica en concordancia con la intensidad y duración de la disciplina deportiva, asintomáticos, con función sistólica y diastólica normales, sin movimiento anterior sistólico mitral, sin obstrucción del tracto de salida del ventrículo izquierdo ni historia familiar de la enfermedad. Fueron comparados con 25 pacientes (grupo B) con $\mathrm{MCH}$ no obstructiva provenientes de otro centro. Encontraron que los atletas tenían un diámetro del ventrículo izquierdo significativamente mayor que el grupo B (60 vs 45 $\mathrm{mm}$ ), de la raíz de aorta (34 vs $30 \mathrm{~mm}$ ) y de la aurícula izquierda (42 vs $33 \mathrm{~mm}$ ), compatibles con sobrecarga de volumen. Si el diámetro del ventrículo izquierdo era < $54 \mathrm{~mm}$, se trataba de una $\mathrm{MCH}$. Si la aurícula izquierda era $>40 \mathrm{~mm}$, era casi siempre de un deportista. El Doppler tisular también permitió esta caracterización ${ }^{(25)}$.

Los atletas afroamericanos, además de las particularidades del ECG ya mencionadas, presentan mayor hipertrofia del ventrículo izquierdo. En un estudio comparativo entre deportistas afroamericanos y caucásicos se encontró que los primeros tenían un espesor parietal medio de $11,3 \mathrm{vs} 10 \mathrm{~mm}$. El $18 \%$ de los afroamericanos y solo $4 \%$ de los caucásicos tenían espesor mayor de $12 \mathrm{~mm}$ y $3 \%$ un espesor mayor de $15 \mathrm{~mm}$ contra ninguno, respectivamente. Nuevamente, los que tenían mayor espesor tenían también dilatación y función sistólica y diastólica normales ${ }^{(26)}$.

\section{Habilitación}

Cuando se encuentra una anormalidad CV o en otro sistema, surge la pregunta de si esa persona puede realizar ejercicio físico y en qué medida. Es imposible establecer un criterio general, ya que el riesgo relativo de las distintas enfermedades también es diferente. Hay enfermedades para las cuales está recomendado realizar cierto grado de ejercicio, y 
otras cuyos eventos o complicaciones se presentan en el reposo, como el síndrome de Brugada. Esta dificultad ha sido motivo de diversos consensos y publicaciones ${ }^{(27-29)}$.

Todas las recomendaciones tienen como referencia la clasificación de los deportes en tres categorías, de acuerdo con el grado del componente aeróbico y anaeróbico antes mencionado. Es conveniente tener accesibles las tablas si uno se desempeña habitualmente en el manejo de esta población.

No es correcto el criterio general de proscribir la actividad deportiva, ya que se puede estar afectando seriamente la calidad de vida de las personas sin un fundamento científico que lo avale.

\section{Conclusiones}

Aunque la evidencia existente sobre este tópico es extremadamente útil, debemos aplicarla en personas y situaciones muy variadas, que a veces difieren de las poblaciones estudiadas originalmente. Es muy importante incorporar datos nacionales que nos permitan una aproximación adecuada al perfil de nuestros deportistas. Sin dudas, la recolección de datos propios proporcionará herramientas para brindar una mejor calidad de atención y optimizar el uso de los recursos.

\section{Alejandro Cuesta, \\ https://orcid.org/0000-0003-1315-5581 \\ Geraldine Rodríguez Estula, \\ https://orcid.org/0000-0001-6326-524X \\ Sergio Giovanetti, \\ https://orcid.org/ 0000-0003-0574-3658}

Este artículo fue aceptado para su publicación por: Editora jefa anterior Dra. María del Pilar Aguilar Passano.

\section{Bibliografía}

1. Peidro, R, Angelino A, Franchella J, Gagliari J, Saglietti JH, Brion G. Consenso corazón y deporte. Comité de Cardiología del Deporte del Consejo de Ergometría y Rehabilitación Cardiovascular de la Sociedad Argentina de Cardiología. Rev Argent Cardiol. 2007;75 Supl 4:S1-29.

2. Peidro R. Cardiología del deporte El corazón del deportista. Hallazgos clínicos, electrocardiográficos y ecocardiográficos. Rev Argent Cardiol. 2003;71(2): 126-37.

3. Mitchell JH, Haskell WL, Raven PB. Classification of sports. J Am Coll Cardiol. 1994;24(4):864-6. doi: 10.1016/0735-1097(94)90841-9.

4. Sztajzel J, Jung M, Sievert K, Bayes De Luna A. Cardiac autonomic profile in different sports discipli- nes during all-day activity. J Sports Med Phys Fitness. 2008;48(4):495-501.

5. D'Souza A, Bucchi A, Johnsen AB, Logantha SJ, Monfredi O, Yanni J, et al. Exercise training reduces resting heart rate via downregulation of the funny channel HCN4. Nat Commun. 2014 May [consulta 20 Ago 2020];5:[aprox.12p.]. Disponible en: https://www.ncbi.nlm.nih.gov/pmc/articles/PMC4024745/pdf/ncomms4775.pdf

6. Lewis S, Nylander E, Gad P, Aertog N. Non autonomic component in bradycardia of endurance trained men at rest and during exercise. Acta Physiol Scand. 1980;109(3):297-305. doi: 10.1111/j.17481716.1980.tb06600.x

7. Chapman JH. Profound sinus bradycardia in the athletic heart syndrome. J Sports Med Phys Fitness 1982;22:45-8.

8. Boraita Pérez A, Serratosa Fernández L. El corazón del deportista: hallazgos electrocardiográficos más frecuentes. Rev Esp Cardiol.1998;51(5):356-68.

9. Meytes I, Kaplinsky E, Yahini JH, Hanne-Paparo N, Neufeld HN. Wenckebach A-V block: a frequent feature following heavy physical training. Am Heart J. 1975;90(4):426-30. doi: 10.1016/0002-8703 (75)90421-4.

10. Viitasalo MT, Kala R, Eisalo A. Ambulatory electrocardiographic recording in endurance athletes. $\mathrm{Br}$ Heart J. 1982;47(3):213-20. doi: 10.1136/hrt.47.3. 213

11. Cooper JP, Fraser AG, Penny WJ. Reversibility and benign recurrence of complete heart block in athletes. Int J Cardiol. 1992;35(1):118-20. doi: 10.1016/ 0167-5273(92)90064-a

12. Chou T-C. Right bundle branch block. En: Chou T-C. Electrocardiography in Clinical Practice: Adult and Pediatric. 4a ed. Philadelphia: W.B. Saunders. 1996. Cap.7.

13. Sharma S, Whyte G, Elliott P, Padula M, Kaushal R, Mahon N, et.al. Electrocardiographic changes in 1000 highly trained junior elite athletes. Br J Sports Med. 1999;33(5):319-24. doi: 10.1136/bjsm. 33.5.319

14. Drezner JA, Fischbach P, Froelicher V, Marek J, Pelliccia A, Prutkin JM, et.al. Normal electrocardiographic findings: recognising physiological adaptations in athletes. Br J Sports Med. 2013;47(3):125-36. doi: 10.1136/bjsports-2012-092068

15. Perez MV, Friday K, Froelicher V. Semantic confusion: the case of early repolarization and the $\mathrm{J}$ point. Am J Med. 2012;125(9):843-4. doi: 10.1016/ j.amjmed.2011.08.024

16. Gussak I, Antzelevitch C. Early repolarization syndrome: a decade of progress. J Electrocardiol. 2013;46(2):110-3. doi: 10.1016/j.jelectrocard.2012. 12.002 
17. Drezner JA, Ackerman MJ, Anderson J, Ashley E, Asplund CA, Baggish AL, et al. Electrocardiographic interpretation in athletes: the 'Seattle criteria'. Br J Sports Med. 2013;47(3):122-4. doi: 10.1136/ bjsports-2012-092067

18. Corrado D, Pelliccia A, Heidbuchel H, Sharma S, Link M, Basso C, et al. Recommendations for interpretation of 12-lead electrocardiogram in the athlete. Eur Heart J. 2010;31(2):243-59. doi: 10.1093/ eurheartj/ehp473

19. Corrado D, Pelliccia A, Bjørnstad HH, Vanhees L, Biffi A, Borjesson M, et al. Cardiovascular pre-participation screening of young competitive athletes for prevention of sudden death: proposal for a common European protocol. Consensus Statement of the Study Group of Sport Cardiology of the Working Group of Cardiac Rehabilitation and Exercise Physiology and the Working Group of Myocardial and Pericardial Diseases of the European Society of Cardiology. Eur Heart J. 2005;26(5):516-24. doi 10.1093/eurheartj/ehi108

20. Brosnan M, La Gerche A, Kalman J, Lo W, Fallon K, MacIsaac A, et al. The Seattle Criteria increase the specificity of preparticipation ECG screening among elite athletes. Br J Sports Med. 2014;48(15):1144-50. doi: 10.1136/bjsports-2013-092420

21. Sheikh N, Papadakis M, Ghani S, Zaidi A, Gati S, Adami PE, et al. Comparison of electrocardiographic criteria for the detection of cardiac abnormalities in elite black and white athletes. Circulation 2014;129(16):1637-49. doi: 10.1161/CIRCULATION AHA 113.006179

22. Corrado D, Basso C, Pavei A, Michieli P, Schiavon M, Thiene G. Trends in sudden cardiovascular death in young competitive athletes after implementation of a preparticipation screening program. JAMA 2006; 296:1593-601. doi: 10.1001/jama.296. 13.1593

23. Pluim BM, Zwinderman AH, van der Laarse A, van der Wall E. The athlete.s heart. A meta-analy- sis of cardiac structure and function. Circulation 2000;101:336-44. doi: 10.1161/01.cir.101.3.336

24. Spirito P, Pelliccia A, Proschan MA, Granata M, Spataro A, Bellone P, et al. Morphology of the "athlete's heart" assessed by echocardiography in 947 elite athletes representing 27 sports. Am J Cardiol. 1994;74(8):802-6. doi: 10.1016/0002-9149(94) 90439-1

25. Caselli S, Maron MS, Urbano-Moral JA, Pandian NG, Maron BJ, Pelliccia A. Differentiating left ventricular hypertrophy in athletes from that in patients with hypertrophic cardiomyopathy. Am J Cardiol. 2014;114(9):1383-9. doi: 10.1016/j.amjcard. 2014.07.070

26. Basavarajaiah S, Boraita A, Whyte G, Wilson M, Carby L, Shah A, et al. Ethnic differences in left ventricular remodeling in highly-trained athletes relevance to differentiating physiologic left ventricular hypertrophy from hypertrophic cardiomyopathy. J Am Coll Cardiol. 2008;51(23):2256-62. doi:10.1016/ j.jacc.2007.12.061

27. Maron B, Zipes D. 36th Bethesda Conference: eligibility recommendations for competitive athletes with cardiovascular abnormalities. J Am Coll Cardiol. 2005; 45(8):1318-75.doi: 10.1016/j.jacc.2005.02.006

28. Pelliccia A, Solberg EE, Papadakis M, Adami PE, Biffi A, Caselli S, et al. Recommendations for participation in competitive and leisuretime sport in athletes with cardiomyopathies, myocarditis, and pericarditis: position statement of the Sport Cardiology Section of the European Association of Preventive Cardiology (EAPC). Eur Heart J. 2019;40(1):19-33. doi: 10.1093/eurheartj/ehy730

29. Pelliccia A, Zipes DP, Maron BJ. Bethesda Conference \#36 and the European Society of Cardiology Consensus Recommendations revisited a comparison of U.S. and European criteria for eligibility and disqualification of competitive athletes with cardiovascular abnormalities. J Am Coll Cardiol. 2008; 52(24):1990-6. doi: 10.1016/j.jacc.2008.08.055 